\title{
Revealing structural dynamics of proteins with time-resolved x-ray liquidography Hyotcherl lhee ${ }^{1}$ \\ ${ }^{1}$ Dept of Chemistry, Inst for Basic Science KAIST \\ ihee57@gmail.com
}

Time-resolved x-ray scattering has now been fully established as a powerful method to investigate molecular structural dynamics in solution [1-10]. We have employed the technique to study structural dynamics of a wide variety of small molecules including diatomic molecules, haloalkanes, organometallic complexes over timescales from femtoseconds to milliseconds. Moreover, the technique has been applied to protein molecules to unveil structural dynamics of proteins in solution. One of the representative experimental methods commonly used for studying structural dynamics of proteins is time-resolved x-ray crystallography. Time-resolved x-ray crystallography can determine atomic-resolution structures of proteins, but has been applied to limited proteins due to the stringent prerequisites such as highly-ordered and radiation-resistant single crystals. The problem can be overcome by applying time-resolved x-ray diffraction directly to protein solutions rather than protein single crystals. To emphasize that structural information can be obtained from the liquid phase, this time-resolved x-ray solution scattering technique is named time-resolved x-ray liquidography (TRXL) in analogy to time-resolved x-ray crystallography where the structural information of reaction intermediates is obtained from the crystalline phase. We will present our recent results including the achievement of femtosecond TRXL using an X-ray free-electron laser. We investigated ultrafast structural dynamics of homodimeric hemoglobin ( $\mathrm{HbI})$, which is a model system for studying allosteric structural transition in proteins. From femtosecond TRXL data of HbI, we revealed detained ultrafast structural changes of $\mathrm{HbI}$ involving a coherent motion of the protein body and time-dependent change of electron density in the hydration shell.

References

[1] H. Ihee et al. Science, 309, 1223-1227 (2005).

[2] K. H. Kim et al. J. Am. Chem. Soc. 134, $7001-7008$ (2012).

[3] T. W. Kim et al. J. Am. Chem. Soc. 134, 3145-3153 (2012).

[4] K. H. Kim et al. Nature, 518, 385-389 (2015).

[5] J. G. Kim et al. Acc. Chem. Res. 48, 2200-2208 (2015).

[6] J. G. Kim et al. Struct. Dyn. 3, 023601 (2016).

[7] H. Kim et al. J. Phys. Chem. B, 124, 1550-1556 (2020).

[8] T. W. Kim et al. Proc. Natl. Acad. Sci. 117, 14996-15005 (2020).

[9] J. G. Kim et al. Nature, 582, 520-524 (2020).

[10] M. Choi et al. Chem. Sci. DOI: 10.1039/D1SC01207J, Online publication: 2021-05-10. 